\title{
Curiosity killed the cat: no evidence of an association between cat ownership and psychotic symptoms at ages 13 and 18 years in a UK general population cohort
}

\author{
F. Solmi*, J. F. Hayes, G. Lewis and J. B. Kirkbride \\ Division of Psychiatry, University College London, London, UK
}

Background. Congenital or early life infection with Toxoplasma gondii has been implicated in schizophrenia aetiology. Childhood cat ownership has been hypothesized as an intermediary marker of $T$. gondii infection and, by proxy, as a risk factor for later psychosis. Evidence supporting this hypothesis is, however, limited.

\begin{abstract}
Method. We used birth cohort data from the Avon Longitudinal Study of Parents and Children (ALSPAC) to investigate whether cat ownership in pregnancy and childhood (ages 4 and 10 years) was associated with psychotic experiences (PEs) in early (age 13, N=6705) and late (age 18, N=4676) adolescence, rated from semi-structured interviews. We used logistic regression to examine associations between cat ownership and PEs, adjusting for several sociodemographic and socioeconomic factors, household characteristics and dog ownership. Missing data were handled via multiple imputation.
\end{abstract}

Results. Cat ownership during pregnancy was not associated with PEs at age 13 years [adjusted odds ratio (OR) 1.15, $95 \%$ confidence interval (CI) $0.97-1.35$ ] or 18 years (OR 1.08, 95\% CI 0.86-1.35). Initial univariable evidence that cat ownership at ages 4 and 10 years was associated with PEs at age 13 years did not persist after multivariable adjustment (4 years: OR 1.18, 95\% CI 0.94-1.48; 10 years: OR 1.12, 95\% CI 0.92-1.36). There was no evidence that childhood cat ownership was associated with PEs at age 18 years.

Conclusions. While pregnant women should continue to avoid handling soiled cat litter, given possible T. gondii exposure, our study strongly indicates that cat ownership in pregnancy or early childhood does not confer an increased risk of later adolescent PEs.

Received 5 September 2016; Revised 4 January 2017; Accepted 6 January 2017; First published online 22 February 2017

Key words: ALSPAC, cat ownership, pet ownership, psychosis, psychotic symptoms.

\section{Introduction}

House cats are the primary hosts of Toxoplasma gondii, a protozoan parasite that can infect various warmblooded animals, including humans (Tenter et al. 2000; Webster et al. 2013). Infection can occur in utero or postnatally, via ingestion of either the parasite's oocysts - which might be present in soil, water, or food-or tissue cysts from infected animals (e.g. in raw or undercooked meat). In intermediate hosts (e.g. humans or animals other than cats), the parasite exploits lymphocytes to encroach in muscle tissues and, importantly, the brain, where it can form tissue cysts in neurons, microglia, and astrocytes (Carruthers \& Suzuki, 2007).

\footnotetext{
* Address for correspondence: F. Solmi, Ph.D., UCL Division of Psychiatry, 6th Floor, Wing B, Maple House, 149 Tottenham Court Road, London W1T 7NF, UK.

(Email: francesca.solmi@ucl.ac.uk)
}

Although the evidence is not unequivocal (Sugden et al. 2016), data from several epidemiological, experimental, and animal studies suggests that T. gondii infection may be implicated in the aetiology of psychosis. For example, dopaminergic dysfunction and cognitive impairments-similar to those observed in people with schizophrenia - have been observed in infected rodents (Gaskell et al. 2009; Prandovszky et al. 2011; McConkey et al. 2013) and humans (Kannan \& Pletnikov, 2012); these people may also experience hallucinations during acute infection with the parasite (Sugden et al. 2016). A recent meta-analysis of 38 studies found that compared with controls, people with schizophrenia were nearly three times more likely to be seropositive for $T$. gondii antibodies [odds ratio (OR) 2.71, 95\% confidence interval (CI) 1.933.80] (Torrey et al. 2012). Higher seroprevalence and serointensity of $T$. gondii IgG (but not IgM, an indicator of recent infection) in people with schizophrenia (Cetinkaya et al. 2007) and their mothers (Brown et al.

This is an Open Access article, distributed under the terms of the Creative Commons Attribution licence (http://creativecommons.org/licenses/by/4.0/), which permits unrestricted re-use, distribution, and reproduction in any medium, provided the original work is properly cited. 
2005; Mortensen et al. 2007a) suggest that either early life exposure to the parasite, congenital infection, or transmission of maternal antibodies could alter neurodevelopment of subsequent offspring.

Assuming a causal relationship between T. gondii infection and later psychosis, some researchers have hypothesized that cat ownership should confer an increased risk of psychotic disorders (Torrey \& Yolken, 1995; Yuksel et al. 2010; Torrey et al. 2015). Moreover, this theory has been proposed to explain several epidemiological findings, including higher rates of psychotic disorders in urban populations (with higher cat densities and subsequent possibility for infection) (Torrey \& Yolken, 1995; Torrey et al. 2000, 2015). Nonetheless, robust empirical evidence supporting this theory remains limited. Cat ownership or contact during pregnancy (Kapperud et al. 1996; Cook et al. 2000) and childhood (Taylor et al. 1997) do not appear to be associated with T. gondii infection, although handling soiled cat litter is known to be associated with infection (Kapperud et al. 1996). Epidemiological studies which have reported an association between cat ownership and psychosis (Torrey \& Yolken, 1995; Torrey et al. 2000, 2015; Yuksel et al. 2010), have generally been hindered by notable methodological limitations (Wolf \& Hamilton, 2015), including reliance on case-control designs that are susceptible to recall bias, small ad hoc samples and weak statistical analyses, which have failed to adequately account for confounding or missing data.

To overcome these issues, we sought to test whether prenatal and childhood cat ownership were associated with an increased risk of developing psychotic experiences (PEs) in early and late adolescence, using longitudinal data from the Avon Longitudinal Study of Parents and Children (ALSPAC). PEs in adolescence are an established risk factor for later schizophrenia, particularly with respect to psychotic symptoms which emerge or persist in late adolescence (Poulton et al. 2000; Fisher et al. 2013). Since T. gondii infection is proposed to increase psychosis risk by affecting early life neurodevelopment (Mortensen et al. 2007b), we restricted cat ownership to the prenatal and early childhood period (at age 4 years), although we also performed additional analyses using cat ownership at age 10 years to better align with previous research (Torrey \& Yolken, 1995; Torrey et al. 2000, 2015; Yuksel et al. 2010).

\section{Method}

\section{Sample}

The ALSPAC study invited 16734 pregnant women expected to deliver between 1 April 1991 and 31
April 1992 resident in the former county of Avon in the Southwest of England to participate; of these, 14 541 (87\%) enrolled resulting in 14062 live births and 13988 children alive at age 1 year. The sample was supplemented with 713 additional children (whose mothers were originally eligible for the study) during follow-up between the ages of 7 and 18 years. More details on recruitment, follow-up assessments and time-points have been published elsewhere (Boyd et al. 2012). All mothers gave informed written consent prior to recruitment and the ALSPAC Ethics and Law Committee and the Local Research Ethics Committees gave ethical approval for this study.

In this study we included children with complete data on PEs at ages $13(N=6705)$ and $18(N=4676)$ years. For twin pairs $(N=87,2.6 \%$ of the sample at age 12 , and $N=42,1.8 \%$ at age 18 ), we excluded one sibling (sibling ' $\mathrm{B}$ ') to avoid biasing estimates due to shared genetic and environmental exposures; there is no evidence that, in twins, birth order is related to schizophrenia risk (Onstad et al. 1992; Kleinhaus et al. 2008).

\section{Exposure variable}

Information on pet ownership was reported by mothers via postal questionnaires during pregnancy, and subsequently when their child was aged 8, 21, 31 and 47 months. In addition to cat ownership, mothers were asked about the number and type of other pets owned, including: dogs, rabbits, rodents, birds (all waves), and tortoises and fish (from 21 months). From these questions we created two primary binary exposure variables, indicating whether the mother owned a cat (yes/no): (i) in pregnancy; and (ii) at child's age of 47 months ( $\sim 4$ years). As a secondary exposure, we employed cat ownership at 10 years (122 months) in order to create an exposure variable comparable with those used in previous studies (Torrey \& Yolken, 1995; Torrey et al. 2000, 2015). We did not test whether a dose-response effect existed between duration of cat ownership and psychotic symptoms, since the majority $(89 \%)$ of children who reported cat ownership at age 4 years also owned one at ages 8, 21, and 31 months, as reported by their mothers.

\section{Outcome variables}

At approximately ages 13 and 18 years, children attended clinic visits where they were administered the psychotic-like symptoms interview (PLIKSi), a semi-structured interviewer-rated screening assessment for PEs. The PLIKSi contains six questions on unusual experiences (i.e. derealization, depersonalization, self-unfamiliarity, dysmorphophobia, partial 
object perception, and other perceptual abnormalities) followed by 12 questions adapted from the Diagnostic Interview Schedule for Children version IV (DISC-IV; Shaffer et al. 2000) and the Schedule for Clinical Assessment in Neuropsychiatry (SCAN; WHO, 1994). These are aimed at assessing the presence of delusions (being spied on, persecuted, having thoughts read, reference, control, grandiose ability, and other delusions), hallucinations (visual and auditory), and intrusive thoughts (thought broadcasting, insertion and withdrawal) (Horwood et al. 2008). Total scores were recoded into a binary variable indicating the absence, or the suspected/definite presence of symptoms, consistent with previous investigations of PEs in ALSPAC (Horwood et al. 2008; Dorrington et al. 2014). Children whose psychotic symptoms could have been attributed to fever or sleep problems were coded as not having the outcome (Zammit et al. 2008, 2009; Dorrington et al. 2014).

\section{Other variables}

We employed causal diagrams, known as directed acyclic graphs (DAGs; Textor \& Liśkiewicz, 2011), to identify variables which could confound the association between cat ownership and psychotic symptoms. We modelled hypothesized associations between a broad initial set of potential child- and mother-based variables, cat ownership in either pregnancy or childhood and PEs using the DAGitty webbased software (Textor et al. 2011). Our DAGs (Supplementary Figs S1 and S2) suggested that it was inappropriate to control for some of these variables, either because they did not meet criteria for confounding (e.g. child gender, stressful life events, maternal depression, pet ownership other than dogs), or because adjustment for other variables (e.g. paternal age, dog ownership in pregnancy) provided sufficient control for any other causal paths (e.g. maternal age, dog ownership at age 4 years). From our DAGs we were able to identify the minimal sufficient number of confounders of the relationship between exposure to cat ownership in pregnancy and childhood and PEs at ages 13 and 18 years. These included: child ethnicity (white/non-whiteincluding Black African, Black Caribbean, Other Black, Indian, Pakistani, Bangladeshi, Chinese, Other, mixed); paternal age (at the time of mother's pregnancy); maternal marital status in pregnancy (single, separated, divorced, or widowed/married); highest maternal academic education in pregnancy (vocational course/secondary schooling/university degree or higher); maternal social class (manual $v$. non-manual profession); number of house moves up to age 47 months ( $\sim 4$ years); housing type (detached, semi- detached, terraced/flat, other); household crowding index (range 0-1); and dog ownership in pregnancy.

The ALSPAC website contains details of all the data that is available through a fully searchable data dictionary (available at: http://www.bris.ac.uk/alspac/ researchers/data-access/data-dictionary/).

\section{Statistical analyses}

We employed cross-tabulations (with $\chi^{2}$ tests) and ANOVAs to (i) investigate the presence of selective attrition in the sample, by comparing children with and without missing outcome data on exposure and confounding variables; (ii) describe the sample and test for differences in the distribution of exposure and confounding variables across outcome levels. We then fitted univariable and multivariable logistic regression models adjusting for all variables identified as relevant confounders using DAGs (Supplementary Figs S1 and S2), i.e.: dog ownership in pregnancy; housing type; household crowding; maternal education, social class, and marital status; paternal age; number of house moves. When the studied exposure was cat ownership in childhood, we further adjusted for maternal cat ownership in pregnancy.

Since our primary hypothesis was a null association between cat ownership and psychotic symptoms, we conducted power calculations, assuming an alpha of 0.05 to determine the effect size we could reasonably expect to detect, had it existed, given our sample sizes. Our sample had over $90 \%$ power to detect an OR of 1.25 based on the observed exposure distribution and sample sizes.

\section{Missing data}

Analyses were based on participants with complete data at age $13(N=6705)$ and $18(N=4676)$ years. Missing main exposures and covariate data varied between $0.04 \%$ (pregnancy) to $34.08 \%$ (age 4 years), and $0.02 \%$ (pregnancy) to $33.58 \%$ (age 4 years) at each time point, respectively (Supplementary Table S1).

We used multiple imputation with chained equations (MICE) and the Stata ice command (Royston \& White, 2011) to impute missing exposure and covariate data, including all observed exposure, covariate and outcome data into our multiple imputation (MI) routine, in addition to several auxiliary variables which could provide information about missing values. These included: gender; stressful life events; maternal depression in pregnancy; other pet (rabbit, rodents, turtles, birds, fish) ownership in pregnancy and age 4 years; two measures of depressive symptoms at ages 12 and 18 years assessed via self-reported with the short moods and feelings questionnaire (SMFQ; 
Angold et al. 1995); a continuous measure of IQ assessed during a clinical assessment at age 8 years using the Wechsler Intelligence Scale for Children third edition (WISC-3); and a measure of family income at child's age of 33 months. We also included a variable indicating maternal history of schizophrenia at birth in light of the known genetic heritability of psychosis (Lichtenstein et al. 2009). We imputed 100 datasets using linear, logistic, ordinal logistic, and multinomial logistic models according to the nature of the variables whose missing values had to be imputed. As sensitivity analyses we examined the association between cat ownership and adolescent PEs in (i) a complete case analysis, to assess any possible bias introduced when failing to account for missing data, and (ii) using data on the full ALSPAC sample $(N=15023)$ with multiple imputation on those missing outcome data, to assess possible biases introduced by our main choice of multiple imputation.

All analyses were performed using Stata v. 13 (StataCorp, 2013).

\section{Results}

\section{Missing data}

Children with missing outcome data at ages 13 and 18 years were more likely to be boys, from a non-white ethnic background, to live in more crowded houses, and have experienced a stressful life event by age 4 years. Children with missing data were also more likely to have younger parents, and a mother who had suffered from probable depression in pregnancy, was less well educated, not married, from a manual occupation, and who had moved house more frequently. At ages 13 and 18 years, children whose mother had owned a cat in pregnancy were less likely to have missing data, although children whose mother had owned a cat in their childhood were more likely to have missing outcome data at age 13 years. Participants with missing outcome data at either age were more likely to have a mother who owned a dog, a bird, a rabbit, or rodent in pregnancy or during childhood (Supplementary Table S1).

\section{Sample characteristics}

A total of 6705 and 4676 children had complete data on psychotic symptoms at ages 13 and 18 years, respectively, and were therefore included in the analyses. At both ages, the majority of each sample was of female gender, white ethnicity, had a mother who had completed at least A-levels, was married, non-manual profession, and had moved house $<3$ times during the 4 years prior to pregnancy and age 4 years of the child (Table 1) In both samples, around one third of mothers owned a cat during pregnancy, and at 4 and 10 years (Table 1). Among children who owned a cat at age 4 years, between $79 \%$ and $89 \%$ also owned a cat at previous waves of data collection (i.e. at ages 8,21 , and 33 months). Among those who owned a cat at age 10 years, between $62 \%$ and $86 \%$ also owned a cat at a previous time point (data available from authors).

At age 13 years, a greater proportion of the sample who reported suspected/definite psychotic symptoms owned a cat in childhood, were girls, lived in flats and in crowded home environments, had moved homes more frequently, and had a mother who was younger, less well educated, and of single marital status (Table 1). Similar patterns were generally observed for the sample at age 18 years (Table 1), although there was no longer any apparent association between PEs and number of house moves, while non-manual social class and non-white ethnicity were associated with experiencing suspected/definite PEs at this age.

\section{Cat ownership and psychotic symptoms}

Cat ownership in pregnancy was not associated with psychotic symptoms at age 13 or 18 years in either univariable (age 13: OR 1.15, 95\% CI 0.97-1.35; age 18; OR 1.08 , 95\% CI $0.86-1.35$ ) or in multivariable (age 13: adjusted OR 1.15, 95\% CI 0.97-1.36; age 18; OR 1.08, 95\% CI 0.85-1.37) models, following multiple imputation (Table 2). Owning a cat at age 4 years was associated with higher odds of having PEs at age 13 years in univariable models (OR 1.23, 95\% CI 1.04-1.46), but this effect was no longer significant after multivariable adjustment (OR 1.18, 95\% CI 0.94-1.48). There was no evidence that cat ownership at age 4 years was associated with PEs at age 18 years (univariable OR 1.11, 95\% CI $0.88-1.40$; adjusted OR 0.97 , 95\% CI $0.71-$ 1.31). These patterns were similar with respect to cat ownership at age 10 years, with no apparent association with $\mathrm{PEs}$ at age 13 years (OR 1.12, 95\% CI 0.92-1.36) or 18 years (OR 1.08, 95\% CI 0.82-1.45) after multivariable adjustment (Table 2).

\section{Sensitivity analyses}

In complete case analyses cat ownership in pregnancy was associated with higher odds of PEs at age 13 years in all models (univariable OR 1.31, 95\% CI 1.04-1.65; adjusted OR 1.34, 95\% CI 1.06-1.69), as was cat ownership at age 4 years (univariable OR 1.44, 95\% CI 1.13-1.83; adjusted OR 1.47, 95\% CI 1.01-2.13). Cat ownership at age 10 years was only associated with PEs at age 13 years in univariable models (OR 1.30, 95\% CI 1.02-1.66), but not in adjusted models. We found no evidence of an association between cat ownership and PEs at age 18 years (Supplementary Table S3). 
Table 1. Sample characteristics

\begin{tabular}{|c|c|c|c|c|c|c|c|c|}
\hline \multirow[b]{2}{*}{ Variables } & \multicolumn{4}{|c|}{ Psychotic experiences age 13 years } & \multicolumn{4}{|c|}{ Psychotic experiences age 18 years } \\
\hline & $\begin{array}{l}N(\%) \\
N=6705\end{array}$ & $\begin{array}{l}\text { None, } N(\%) \\
N=5929(88.43 \%)\end{array}$ & $\begin{array}{l}\text { Suspected or definite, } \\
N(\%), N=776(11.57 \%)\end{array}$ & $p\left(\chi^{2}\right)$ & $\begin{array}{l}N(\%) \\
(N=4676)\end{array}$ & $\begin{array}{l}\text { None, } N(\%) \\
N=4306(92.09 \%)\end{array}$ & $\begin{array}{l}\text { Suspected or definite, } \\
N(\%), N=370(7.91 \%)\end{array}$ & $p\left(\chi^{2}\right)$ \\
\hline Cat ownership in pregnancy (yes) & $1959(31.66)$ & 1719 (31.35) & $240(34.09)$ & 0.14 & $1336(31.17)$ & $1227(31.06)$ & 109 (32.44) & 0.60 \\
\hline Cat ownership at age 4 (yes) & $1620(28.78)$ & $1407(28.21)$ & $213(33.23)$ & 0.008 & $1017(28.54)$ & $1019(28.47)$ & $88(29.33)$ & 0.75 \\
\hline Cat ownership at age 10 (yes) & $1837(31.83)$ & $1611(31.40)$ & $226(35.26)$ & 0.05 & $1232(31.44)$ & $1134(31.22)$ & $98(34.15)$ & 0.30 \\
\hline Dog ownership in pregnancy (yes) & $1380(22.30)$ & 1209 (22.05) & $171(24.29)$ & 0.18 & $880(20.53)$ & $800(20.25)$ & $80(23.81)$ & 0.79 \\
\hline Gender (female) & $3420(51.03)$ & $2995(50.54)$ & $425(54.77)$ & 0.03 & $2639(56.45)$ & $2401(55.77)$ & $238(64.32)$ & 0.001 \\
\hline Ethnicity (Non-white) & $239(3.95)$ & $209(3.90)$ & $30(4.34)$ & 0.58 & $180(4.30)$ & $159(4.11)$ & $21(6.56)$ & 0.04 \\
\hline Social class (manual) & $833(15.62)$ & $728(15.39)$ & $105(17.38)$ & 0.20 & $568(15.22)$ & $507(14.69)$ & $61(21.71)$ & 0.002 \\
\hline Housing type (flat) & $812(13.19)$ & 685 (12.55) & $127(18.14)$ & $<0.0001$ & $562(13.16)$ & 495 (12.57) & $67(20.12)$ & $<0.0001$ \\
\hline Marital status (married) & $5046(81.24)$ & $4523(82.13)$ & $523(74.29)$ & $<0.0001$ & $3516(81.63)$ & $3286(82.73)$ & $230(68.66)$ & $<0.0001$ \\
\hline \multicolumn{9}{|l|}{ Maternal education } \\
\hline Up to A level & $3823(62.28)$ & $3366(61.97)$ & 457 (64.73) & 0.03 & $2628(61.79)$ & $2437(62.17)$ & $191(57.36)$ & $<0.0001$ \\
\hline Degree or higher & $1005(16.37)$ & $914(16.83)$ & $91(12.89)$ & & $822(19.33)$ & $771(19.67)$ & $51(15.32)$ & \\
\hline \multicolumn{9}{|l|}{ Number of house moves } \\
\hline $1-3$ & $1417(29.23)$ & $1245(29.00)$ & $172(31.39)$ & 0.05 & $993(29.24)$ & $912(29.02)$ & $81(32.02)$ & 0.09 \\
\hline$\geqslant 4$ & $629(13.00)$ & $545(12.67)$ & $84(15.33)$ & & $428(12.60)$ & $388(12.34)$ & $40(15.81)$ & \\
\hline \multicolumn{9}{|l|}{ Crowding index } \\
\hline $0.25 \%$ & $1892(31.03)$ & $1685(31.16)$ & $207(29.96)$ & 0.02 & $1278(30.18)$ & $1179(30.20)$ & $99(30.00)$ & $<0.0001$ \\
\hline $0.75 \%$ & $929(15.23)$ & $814(15.05)$ & $115(16.64)$ & & $599(14.15)$ & $532(13.63)$ & $67(20.30)$ & \\
\hline \multirow[t]{2}{*}{$1 \%$} & $241(3.95)$ & $200(3.70)$ & $41(5.93)$ & & $161(3.80)$ & $138(3.53)$ & $23(6.97)$ & \\
\hline & & Mean S.D. & Mean S.D. & & Mean S.D. & Mean S.D. & Mean S.D. & \\
\hline Maternal age, years & $29.18(4.52)$ & $28.79(4.51)$ & $28.19(4.72)$ & 0.001 & $29.42(4.57)$ & $29.03(4.53)$ & $27.69(5.23)$ & $<0.0001$ \\
\hline Paternal age, years & $30.71(6.72)$ & $31.33(5.46)$ & $31.14(5.53)$ & 0.46 & $31.10(6.76)$ & $31.59(5.52)$ & $30.54(5.40)$ & 0.005 \\
\hline
\end{tabular}


Table 2. Univariable and multivariable odds ratios (OR) and 95\% confidence intervals (CI) for the association between maternal cat ownership in pregnancy and between the ages of 8 months and 4 years of the child and psychotic symptoms (suspected or definite v. none) at ages 13 and 18 years ( $N$ includes exposure and confounding variables imputed with multiple imputation with chained equations, $N=100$ imputations)

Psychotic experiences age 13 (suspected or definite $v$. none) $N=6705$

Crude OR $(95 \% \mathrm{CI})$

Adjusted $\mathrm{OR}^{\mathrm{a}}(95 \% \mathrm{CI})$

\begin{tabular}{|c|c|c|}
\hline \multicolumn{3}{|c|}{ Exposure variable } \\
\hline \multicolumn{3}{|c|}{ Cat ownership in pregnancy } \\
\hline No & Ref. & Ref. \\
\hline Yes & $1.15(0.97-1.35)$ & $1.15(0.97-1.36)$ \\
\hline \multicolumn{3}{|c|}{ Cat ownership at age 4} \\
\hline No & Ref. & Ref. \\
\hline Yes & $1.23(1.04-1.46)^{* *}$ & $1.18(0.94-1.48)$ \\
\hline \multicolumn{3}{|c|}{ Cat ownership at age 10} \\
\hline No & Ref. & Ref. \\
\hline Yes & $1.19(1.00-1.41)^{* *}$ & $1.12(0.92-1.36)$ \\
\hline
\end{tabular}

Psychotic experiences age 18 (suspected or definite $v$. none) $N=4676$

Crude OR $(95 \% \mathrm{CI}) \quad$ Adjusted $\mathrm{OR}^{\mathrm{a}}(95 \% \mathrm{CI})$

Cat ownership in pregnancy

No Ref

Yes
at ownership at age 4

No

Yes

Cat ownership at age 10

No

Yes
1.08 (0.86-1.35)

Ref.

1.11 (0.88-1.40)

Ref.

1.15 (0.89-1.48)
Ref.

1.08 (0.85-1.37)

Ref.

0.97 (0.71-1.31)

Ref.

$1.08(0.82-1.45)$

${ }^{* *} p<0.05$.

${ }^{a}$ Model of cat ownership in pregnancy is adjusted for child ethnicity; maternal education, marital status, and social class; paternal age; number of house moves until age 4, type of house, crowding index. Models of cat ownership in at ages 4 and 10 years are further adjusted for cat ownership in pregnancy.

Next, we used the fully imputed dataset to examine the association between cat ownership and adolescent PEs on the whole ALSPAC cohort $(N=15023)$. The pattern of these results (Supplementary Table S4) was very similar to the magnitude, direction and general lack of association between cat ownership and adolescent PEs reported in our main results based on imputation of exposures and confounders (Table 2). Together these sensitivity analyses suggested that complete case analyses may lead to biased risk estimates, while our choice of MI routine did not substantially bias our results.

\section{Discussion}

We found no evidence that cat ownership in pregnancy or childhood was associated with PEs in early and late adolescence using prospectively collected data from a large population-based cohort, following control for several confounders and methods that investigate the likely impact of missing data.

Our findings in relation to PEs are not consistent with the existing literature that has studied cat ownership in people with schizophrenia (Torrey \& Yolken, 1995; Torrey et al. 2000, 2015; Yuksel et al. 2010). We suggest that several methodological differences between our study and other investigations, including previous reliance on small, retrospective, convenience samples, may explain the discrepancy.

Our study was based on PEs in early and late adolescence, unlike other studies which were based on a clinical diagnosis of schizophrenia (Torrey \& Yolken, 1995; Torrey et al. 2000, 2015; Yuksel et al. 2010). One possible explanation of our null findings is that cat ownership does not affect the population expression of psychosis (i.e. does not shift the continuum), but operates only to increase risk of threshold symptoms for clinical disorder. We consider this explanation unlikely, given 
that: psychotic symptoms in late childhood and adolescence predict onset of non-affective psychosis and other psychopathology (Poulton et al. 2000; Laurens et al. 2007; Kelleher et al. 2012; Fisher et al. 2013) and psychotic symptoms in late adolescence are better predictors of future psychopathology. In line with other literature (Kelleher et al. 2012), we observed a decline in the prevalence of PEs between ages 13 and 18 years. If symptoms in later adolescence therefore provide a greater indication of later clinical disorder (Poulton et al. 2000; Fisher et al. 2013) then our results at age 18 (where there was no evidence of any association between cat ownership and PEs) may have the strongest implications for likely effects of early life cat ownership on clinical disorder.

Unlike previous studies which investigated cat ownership up until ages 10 or 13 years (Torrey \& Yolken, 1995; Torrey et al. 2000, 2015; Yuksel et al. 2010), we restricted our main exposure variables to cat ownership during potentially sensitive windows of neurodevelopment, namely pregnancy and at age 4 years (47 months). We included cat ownership at age 10 years as a secondary exposure, consistent with previous literature, but our results mirrored those found at age 4 years, generally indicating an absence of association. Our study was sufficiently powered to detect effect sizes previously observed in the schizophrenia literature with respect to cat ownership (ORs $\geqslant 1.25$ ) (Torrey \& Yolken, 1995; Torrey et al. 2000, 2015; Yuksel et al. 2010), as evidenced by the initial univariable associations we only observed between childhood exposure and PEs at age 13 years. While our study would have had less power to detect smaller ORs, including those we observed between 1.04 and 1.15, any such small effects, if true, would not warrant particular public mental health attention.

Previous reports of positive associations between cat ownership and schizophrenia may therefore have been attributable to Type I error, particularly given the small sample sizes and lack of control for confounders inherent to some studies. We adjusted for several, theoretically-informed confounders, including ethnicity (Westgarth et al. 2010; Kirkbride et al. 2012), maternal academic achievement and social class (Mulvany et al. 2001; Werner et al. 2007; Westgarth et al. 2010), and parental age (Sipos et al. 2004; Lopez-Castroman et al. 2010; Westgarth et al. 2010; Petersen et al. 2011). We also adjusted for number of house moves in light of evidence of an association between residential mobility and PEs (Singh et al. 2014), crowding index and housing type as a proxies for both social class and greater possibility of contact with T. gondii-contaminated litter, and dog ownership as a possible confounder of the association between T. gondii infection (given an increased likelihood to contaminated soils outdoors) and psychosis risk.
Earlier studies also relied on retrospective recall, and hence the potential of recall bias, of cat exposure and did not distinguish between ownership in infancy $v$. later childhood, making it impossible to attribute risk to specific periods of cat ownership over the early life-course.

Finally, we employed multiple imputation techniques in order to account for missing data, which could have otherwise biased our results. Consistent with guidelines (Sterne et al. 2009), we included all known exposure, outcome, covariate and auxiliary variables in $\mathrm{MI}$ as well as additional variables which had been previously found to be associated with PEs in this sample. Comparing our results with those from complete case analyses suggests that selective participation may potentially bias estimates, and could therefore explain previous positive findings in the literature (Torrey \& Yolken, 1995; Torrey et al. 2000, 2015). This hypothesis is further supported by our results using the fully-imputed sample, where no significant associations were found in line with our main findings.

In conclusion, there is good evidence to support an association between $T$. gondii infection and later risk of experiencing psychosis, and this research is consistent with possible inflammatory causes of schizophrenia and other psychotic disorders. From a public health perspective, however, it is perhaps reassuringly that data from our prospective longitudinal study were not consistent with the hypothesis that cat ownership in pregnancy or early childhood is a risk factor for later psychosis.

\section{Supplementary material}

The supplementary material for this article can be found at https://doi.org/10.1017/S0033291717000125.

\section{Acknowledgements}

Dr James Kirkbride and Dr Francesca Solmi are supported by a Sir Henry Dale Fellowship to J.B.K., jointly funded by the Wellcome Trust and the Royal Society (grant no. 101272/Z/13/Z). The UK Medical Research Council and the Wellcome Trust (grant ref. 102215/2/ 13/2; grant ref. GR072043MA MRC G0701503) and the University of Bristol provide core support for ALSPAC. This publication is the work of the authors and will serve as guarantors for the contents of this paper. We are extremely grateful to all the families who took part in this study, the midwives for their help in recruiting them, and the whole ALSPAC team, which includes interviewers, computer and laboratory technicians, clerical workers, research scientists, volunteers, managers, receptionists and nurses. 


\section{Declaration of Interest}

F.S., J.F.H., G.L., and J.B.K. currently own or have owned cats (F.S., J.F.H., and J.B.K. $N=1$; G.L. $N=2$ ), but declare that the latter did not have a role in the formulation of the study hypothesis.

\section{References}

Angold A, Costello EJ, Messer SC, Pickles A, Winder F, Silver D (1995). Development of a short questionnaire for use in epidemiological studies of depression in children and adolescents. International Journal of Methods in Psychiatric Research 5, 237-249.

Boyd A, Golding J, Macleod J, Lawlor D, Fraser A, Henderson J, Molloy L, Ness A, Ring S, Davey Smith G (2012). Cohort Profile: The 'Children of the 90s' - the index offspring of the Avon Longitudinal Study of Parents and Children. International Journal of Epidemiology 42, 111-127.

Brown AS, Schaefer CA, Quesenberry CP, Liu L, Babulas VP, Susser ES (2005). Maternal exposure to toxoplasmosis and risk of schizophrenia in adult offspring. American Journal of Psychiatry 162, 767-773.

Carruthers VB, Suzuki Y (2007). Effects of Toxoplasma gondii infection on the brain. Schizophrenia Bulletin 33, 745-751.

Cetinkaya Z, Yazar S, Gecici O, Namli MN (2007). Anti-Toxoplasma gondii antibodies in patients with schizophrenia - preliminary findings in a Turkish sample. Schizophrenia Bulletin 33, 789-791.

Cook AJ, Gilbert RE, Buffolano W, Zufferey J, Petersen E, Jenum PA, Foulon W, Semprini AE, Dunn DT (2000). Sources of toxoplasma infection in pregnant women: European multicentre case-control study. European Research Network on Congenital Toxoplasmosis. British Medical Journal (Clinical Research Edition) 321, 142-147.

Dorrington S, Zammit S, Asher L, Evans J, Heron J, Lewis G (2014). Perinatal maternal life events and psychotic experiences in children at twelve years in a birth cohort study. Schizophrenia Research 152, 158-163.

Fisher HL, Caspi A, Poulton R, Meier MH, Houts R, Harrington H, Arseneault L, Moffitt TE (2013). Specificity of childhood psychotic symptoms for predicting schizophrenia by 38 years of age: a birth cohort study. Psychological Medicine 43, 2077-2086.

Gaskell EA, Smith JE, Pinney JW, Westhead DR, McConkey GA (2009). A unique dual activity amino acid hydroxylase in Toxoplasma gondii. PLoS ONE 4, e4801.

Horwood J, Salvi G, Thomas K, Duffy L, Gunnell D, Hollis C, Lewis G, Menezes P, Thompson A, Wolke D, Zammit S, Harrison G (2008). IQ and non-clinical psychotic symptoms in 12-year-olds: results from the ALSPAC birth cohort. British Journal of Psychiatry 193, 185-191.

Kannan G, Pletnikov MV (2012). Toxoplasma gondii and cognitive deficits in schizophrenia: an animal model perspective. Schizophrenia Bulletin 38, 1155-1161.

Kapperud G, Jenum PA, Stray-Pedersen B, Melby KK, Eskild A, Eng J (1996). Risk factors for Toxoplasma gondii infection in pregnancy: results of a prospective case-control study in Norway. American Journal of Epidemiology 144, 405-412.

Kelleher I, Keeley H, Corcoran P, Lynch F, Fitzpatrick C, Devlin N, Molloy C, Roddy S, Clarke MC, Harley M, Arseneault L, Wasserman C, Carli V, Sarchiapone M, Hoven C, Wasserman D, Cannon M (2012).

Clinicopathological significance of psychotic experiences in non-psychotic young people: evidence from four populationbased studies. British Journal of Psychiatry 201, 26-32.

Kirkbride JB, Errazuriz A, Croudace TJ, Morgan C, Jackson D, Boydell J, Murray RM, Jones PB (2012). Incidence of schizophrenia and other psychoses in England, 1950-2009: a systematic review and meta-analyses. PLoS ONE 7, e31660.

Kleinhaus K, Harlap S, Perrin MC, Manor O, CalderonMargalit R, Friedlander Y, Malaspina D (2008). Twin pregnancy and the risk of schizophrenia. Schizophrenia Research 105, 197-200.

Laurens KR, Hodgins S, Maughan B, Murray RM, Rutter ML, Taylor EA (2007). Community screening for psychoticlike experiences and other putative antecedents of schizophrenia in children aged 9-12 years. Schizophrenia Research 90, 130-146.

Lichtenstein P, Yip BH, Björk C, Pawitan Y, Cannon TD, Sullivan PF, Hultman CM (2009). Common genetic determinants of schizophrenia and bipolar disorder in Swedish families: a population-based study. Lancet 373, 234-239.

Lopez-Castroman J, Gómez DD, Belloso JJC, FernandezNavarro P, Perez-Rodriguez MM, Villamor IB, Navarrete FF, Ginestar CM, Currier D, Torres MR, Navio-Acosta M, Saiz-Ruiz J, Jimenez-Arriero MA, Baca-Garcia E (2010). Differences in maternal and paternal age between schizophrenia and other psychiatric disorders. Schizophrenia Research 116, 184-90.

McConkey GA, Martin HL, Bristow GC, Webster JP (2013). Toxoplasma gondii infection and behaviour - location, location, location? Journal of Experimental Biology 216, 113-119.

Mortensen PB, Nørgaard-Pedersen B, Waltoft BL, Sørensen TL, Hougaard D, Torrey EF, Yolken RH (2007a). Toxoplasma gondii as a risk factor for early-onset schizophrenia: analysis of filter paper blood samples obtained at birth. Biological Psychiatry 61, 688-693.

Mortensen PB, Nørgaard-Pedersen B, Waltoft BL, Sørensen TL, Hougaard D, Yolken RH (2007b). Early infections of Toxoplasma gondii and the later development of schizophrenia. Schizophrenia Bulletin 33, 741-744.

Mulvany F, O'Callaghan E, Takei N, Byrne M, Fearon P, Larkin C, Wiersma D, Giel R, De Jong A, Slooff C, Argyle M, Jones P, Bebbington P, Foerster A, Lewis S, Murray R, Russell A, Aro S, Aro H, Keskimäki I, Warner R, de Girolamo G, Croudace T, Kayne R, Jones P, Harrison G, Geddes J, Lawrie S, Hultman C, Sparén P, Takei N, Murray R, Cnattingius S, Geddes J, Sham P, O'Callaghan E, Takei N, Susser E, Lin S, Wynn S, Wynn A, Doyle W, Saxena S, Majeed A, Jones $M$, Turner R, Wagenfeld M, Hare E, Price J, Slater E, Loebel A, Lieberman J, Alvir J, Mayerhoff D, Geisler S, Szymanski S, Carbone S, Harrigan S, McGorry P, Birtchnell J, Schwartz J, Fennig S, Tanenberg-Karant M, Carlson G, Craig T, Galambos N, 
Mäkikyrö T, Isohanni M, Moring J, Dohrenwend B, Levav I, Shrout P, Schwartz S, Naveh G, Link B, Cooper B, Roberts K, Bosma H, Schrijvers C, Mackenbach J, Clarke M, Brown S, McTigue O, Gervin M, Murphy P, Waddington J (2001). Effect of social class at birth on risk and presentation of schizophrenia: case-control study. British Medical Journal (Clinical Research Edition) 323, 13981401.

Onstad S, Skre I, Torgersen S, Kringlen E (1992).

Birthweight and obstetric complications in schizophrenic twins. Blackwell Publishing Ltd Acta Psychiatrica Scandinavica 85, 70-73.

Petersen L, Mortensen PB, Pedersen CB (2011). Paternal age at birth of first child and risk of schizophrenia. American Psychiatric Publishing Arlington, VA American Journal of Psychiatry 168, 82-88.

Poulton R, Caspi A, Moffitt TE, Cannon M, Murray R, Harrington H (2000). Children's self-reported psychotic symptoms and adult schizophreniform disorder: a 15-year longitudinal study. Archives of General Psychiatry 57, 10531058.

Prandovszky E, Gaskell E, Martin H, Dubey JP, Webster JP, McConkey GA (2011). The neurotropic parasite Toxoplasma gondii increases dopamine metabolism. Public Library of Science PLoS ONE 6, e23866.

Royston P, White IR (2011). Multiple Imputation by Chained Equations (MICE): implementation in Stata. Journal of Statistical Software 45, 1-20.

Shaffer D, Fisher P, Lucas CP, Dulcan MK, Schwab-Stone ME (2000). NIMH Diagnostic Interview Schedule for Children Version IV (NIMH DISC-IV): description, differences from previous versions, and reliability of some common diagnoses. Journal of the American Academy of Child and Adolescent Psychiatry 39, 28-38.

Singh SP, Winsper C, Wolke D, et al. (2014). School mobility and prospective pathways to psychotic-like symptoms in early adolescence: a prospective birth cohort study. Journal of the American Academy of Child and Adolescent Psychiatry 53, 518-27.

Sipos A, Rasmussen F, Harrison G, Tynelius P, Lewis G, Leon DA, Gunnell D (2004). Paternal age and schizophrenia: a population based cohort study. British Medical Journal (Clinical Research Edition) 329, 1070.

StataCorp (2013). Stata Statistical Software: Release 13. StataCorp LP: College Station, TX 13.

Sterne JAC, White IR, Carlin JB, Spratt M, Royston P, Kenward MG, Wood AM, Carpenter JR (2009). Multiple imputation for missing data in epidemiological and clinical research: potential and pitfalls. British Medical Journal (Clinical Research Edition) 338, b2393.

Sugden K, Moffitt TE, Pinto L, Poulton R, Williams BS, Caspi A (2016). Is Toxoplasma gondii infection related to brain and behavior impairments in humans? Evidence from a population-representative birth cohort. Public Library of Science PLOS ONE 11, e0148435.

Taylor MR, Lennon B, Holland CV, Cafferkey M (1997). Community study of toxoplasma antibodies in urban and rural schoolchildren aged 4 to 18 years. Archives of Disease in Childhood 77, 406-409.
Tenter AM, Heckeroth AR, Weiss LM (2000). Toxoplasma gondii: from animals to humans. International Journal for Parasitology 30, 1217-1258.

Textor J, Hardt J, Knüppel S (2011). DAGitty. Epidemiology 22, 745.

Textor J, Liśkiewicz M (2011). Adjustment criteria in causal diagrams: an algorithmic perspective. In Proceedings of the 27th Conference on Uncertainty in Artificial Intelligence, pp. 681-688.

Torrey EF, Bartko JJ, Yolken RH (2012). Toxoplasma gondii and other risk factors for schizophrenia: an update. Schizophrenia Bulletin 38, 642-647.

Torrey EF, Rawlings R, Yolken RH (2000). The antecedents of psychoses: a case-control study of selected risk factors. Schizophrenia Research 46, 17-23.

Torrey EF, Simmons W, Yolken RH (2015). Is childhood cat ownership a risk factor for schizophrenia later in life? Schizophrenia Research 165, 1-2.

Torrey EF, Yolken RH (1995). Could schizophrenia be a viral zoonosis transmitted from house cats? Schizophrenia Bulletin 21, 167-171.

Webster JP, Kaushik M, Bristow GC, McConkey GA (2013). Toxoplasma gondii infection, from predation to schizophrenia: can animal behaviour help us understand human behaviour? Journal of Experimental Biology 216, 99-112.

Werner S, Malaspina D, Rabinowitz J (2007). Socioeconomic status at birth is associated with risk of Schizophrenia: population-based multilevel study. Schizophrenia Bulletin 33, 1373-1378.

Westgarth C, Heron J, Ness AR, Bundred P, Gaskell RM, Coyne KP, German AJ, McCune S, Dawson S (2010). Family pet ownership during childhood: findings from a UK birth cohort and implications for public health research. International Journal of Environmental Research and Public Health 7, 3704-3729.

WHO (1994). SCAN: Schedules for Clinical Assessment in Neuropsychiatry, Version 2.0. Geneva.

Wolf PJ, Hamilton FE (2015). Flawed analyses undermine proposed relationship between childhood cat ownership and schizophrenia. Schizophrenia Research 168, 596.

Yuksel P, Alpay N, Babur C, Bayar R, Saribas S, Karakose AR, Aksoy C, Asian M, Mehmetali S, Kilic S, Balcioglu I, Hamanca O, Dirican A, Kucukbasmaci O, Oner A, Torun MM, Kocazeybek B (2010). The role of latent toxoplasmosis in the aetiopathogenesis of schizophrenia - the risk factor or an indication of a contact with cat? Folia Parasitologica 57, 121-128.

Zammit S, Horwood J, Thompson A, Thomas K, Menezes P, Gunnell D, Hollis C, Wolke D, Lewis G, Harrison G (2008). Investigating if psychosis-like symptoms (PLIKS) are associated with family history of schizophrenia or paternal age in the ALSPAC birth cohort. Schizophrenia Research 104, 279-286.

Zammit S, Thomas K, Thompson A, Horwood J, Menezes P, Gunnell D, Hollis C, Wolke D, Lewis G, Harrison G (2009). Maternal tobacco, cannabis and alcohol use during pregnancy and risk of adolescent psychotic symptoms in offspring. British Journal of Psychiatry 195, 294-300. 